\title{
Condições Necessárias para Problemas de Controle Ótimo Discreto com Restrições Mistas via Formalismo de Dubovitskii-Milyutin
}

\author{
John F. M. Ascona ${ }^{1}$ \\ IMECC/UNICAMP, Campinas, SP \\ Roberto Andreani ${ }^{2}$ \\ IMECC/UNICAMP, Campinas, SP \\ Valeriano A. de Oliveira ${ }^{3}$ \\ IBILCE/UNESP, São José do Rio Preto, SP
}

\begin{abstract}
Resumo Neste trabalho fornecemos condições necessárias não degeneradas de primeira ordem para problemas de controle ótimo discreto com restrições mistas usando uma condição de regularidade combinada do tipo Mangasarian-Fromovitz e posto constante. As condições de otimalidade foram obtidas via formalismo de Dubovitskii-Milyutin.
\end{abstract}

Palavras-chave. Problemas de Controle Ótimo Discreto, Condições não Degeneradas, Princípio do Máximo Discreto, Formalismo de Dubovitskii-Milyutin.

\section{Introdução}

Problemas de controle ótimo discreto (ou problemas de controle ótimo com tempo discreto) aparecem no estudo da simulação de sistemas práticos controlados onde as mudanças no estado e no controle podem acontecer em períodos de tempo. No mundo real, diversos fenômenos acontecem em intervalos discretos, por exemplo, os números das importações e exportações são relatados trimestralmente. Outras aplicações podem ser encontradas por exemplo em [9]. Logo, do ponto de vista prático, o estudo de problemas de controle ótimo discreto (COD) é muito importante. Por outro lado, do ponto de vista teórico, à princípio, pode-se pensar que os problemas COD não são muito relevantes porque constituem um caso particular dos problemas programação matemática. Ainda que os problemas COD possam ser reduzidos a um problema programação matemática, mesmo assim este tipo de problema tem características próprias. Portanto, é razoável esperar que as condições de otimalidade (necessárias ou suficientes) para este tipo de problema também sejam formuladas de uma maneira mais particular. Por esses motivos surge a necessidade de realizar um estudo específico de problemas COD (ver por exemplo [2], [4], [7], [8]).

$\mathrm{Na}$ literatura, os problemas COD foram tratados usando as hipóteses de regularidade tipo Abadie ou MFCQ (ver por exemplo [7], [8]), onde foram consideradas restrições de contorno de igualdade ou restrições de contorno de desigualdade. Além disso, há muitos artigos tanto em programação não linear como em controle ótimo que usam a condição de posto constante (ver por exemplo [1], [5]).

\footnotetext{
${ }^{1}$ fjmatos19@gmail.com.

2 andreani@ime.unicamp.br.

3 valeriano.oliveira@unesp.br.
} 
O principal objetivo deste trabalho é apresentar condições necessárias de otimalidade de primeira ordem para o problema de controle ótimo discreto com restrições mistas (isto é, problemas COD com restrições de estado e de controle). Este tipo de restrições são importantes pois possuem diversas aplicações, por exemplo, o tamanho máximo do crédito bancário factível (controle de investimento) pode depender do estoque de capital atual (variável de estado), ver [6]. Estas condições necessárias de primeira ordem é uma versão discreta do Princípio do Máximo e são obtidas via formalismo de Dubovitskii-Milyutin. Há muitos trabalhos que usaram este formalismo para obter condições de otimalidade, por exemplo em [3] é dado uma interessante prova do Princípio do Máximo de Pontryagim para problemas de controle ótimo com tempo contínuo e em [8] é feito uma prova deste princípio para o tempo discreto, ambos usando o formalismo.

\section{Preliminares}

O formalismo de Dubovitski-Milyutin é um método interessante para a análise de uma extensa variedade de problemas de otimização, elaborados de maneira mais abstrata, ou seja, as funções podem ser definidas em espaços abstratos. Assim, este método pode ser usado para a análise dos mais variados problemas, como por exemplo, Problemas de Controle Ótimo, Problemas de Programação Matemática, entre outros. Este formalismo fornece condições necessárias de otimalidade para o seguinte problema de otimização:

$$
\begin{array}{cl}
\text { Minimizar } & f(x) \\
\text { sujeito a } & x \in Q \cap V,
\end{array}
$$

onde $f: X \rightarrow \mathbb{R}, X$ é um espaço de Banach, $V$ é uma vizinhança da solução ótima, e $Q=$ $\cap_{i=1}^{l+1} Q_{i}$, onde $Q_{i}$, para $i=1, \ldots, l$, tem interior não vazio e são conjuntos dados pelas restrições de desigualdade, $Q_{l+1}$ tem interior vazio e é dado por um sistema de restrições de igualdade.

Observação 2.1. Nosso interesse é trabalhar com problemas do tipo $\left(P_{1}\right)$, mas na teoria geral $X$ pode não ser um espaço de Banach, basta ser um espaço vetorial topológico, e os conjuntos $Q_{i}$ podem não ser dados por sistemas de igualdade e de desigualdade, podem ser restrições abstratas.

A seguir enunciamos alguns resultados importantes, que serão utilizados para a obtenção das condições necessárias de primeira ordem para o problema $\left(P_{1}\right)$ por meio do Formalismo de Dubovitskii-Milyutin. .

Definição 2.1. Dizemos que $d \in X$ é uma direção de descida do funcional $f: X \rightarrow \mathbb{R}$ no ponto $x^{*} \in X$ se existem um escalar $\epsilon^{*}>0$, uma vizinhança $V$ do vetor $d$ e um número $\alpha=$ $\alpha\left(f, x^{*}, d\right), \alpha<0$, tais que para todo $0<\epsilon<\epsilon^{*}$ e qualquer $\bar{d} \in V, f\left(x^{*}+\epsilon \bar{d}\right) \leq f\left(x^{*}\right)+\epsilon \alpha$.

Definição 2.2. Seja $Q \subset X$, com int $Q \neq \emptyset$. Dizemos que $d \in X$ é uma direção factível em relação ao conjunto $Q$ no ponto $x^{*} \in Q$ se existem uma vizinhança $U$ do vetor $d$ e um escalar $\epsilon^{*}>0$ tais que, para todo $\epsilon \in\left(0, \epsilon^{*}\right)$ e todo $\bar{d} \in U, x^{*}+\epsilon \bar{d} \in Q$.

Definição 2.3. Seja $Q \subset X$. Dizemos que um vetor $v \in X$ é uma direção tangente a $Q$ no ponto $x^{*}$ se existem $\epsilon^{*}>0$ e uma função $r:\left(0, \epsilon^{*}\right) \rightarrow X$ tais que para todo $\epsilon \in\left(0, \epsilon^{*}\right)$ temos $x^{*}+\epsilon v+r(\epsilon) \in Q, \quad\|r(\epsilon)\|=o(\epsilon)$.

Observação 2.2. Os conjuntos de direções de descida e factíveis são cones abertos com vértice na origem, enquanto o conjunto de direções tangentes é um cone com vértice na origem que geralmente não é aberto nem fechado. 
Definição 2.4. Seja $K$ um cone em um espaço vetorial topológico $\boldsymbol{Y}$ com vértice na origem. $O$ cone dual $K^{*} \subset \boldsymbol{Y}^{*}$ é definido por $K^{*}=\left\{l \in \boldsymbol{Y}^{*}: l(x) \geq 0\right.$ para todo $\left.x \in K\right\}$, ou seja, $K^{*} e ́$ definido como o conjunto de todos os funcionais lineares contínuos não negativos em $K$, onde $\boldsymbol{Y}^{*}$ é o dual topológico do espaço $Y$ (Note que $K^{*}$ é um cone convexo com vértice na origem).

Teorema 2.1. (Dubovitskii-Milyutin) Sejam $C_{0}$ o cone das direções de descida de $f$ no ponto $x^{*}, C_{i}$ o cone das direções factiveis a $Q_{i}(i=1 \ldots, \ell)$ no ponto $x^{*}$ e $C_{\ell+1}$ o cone das direções tangentes a $Q_{\ell+1}$ no ponto $x^{*}$. Suponha que todos os cones são convexos. Se o funcional $f$ assume um mínimo local em $Q=\cap_{i=1}^{\ell+1} Q_{i}$ no ponto $x^{*} \in Q$, então existem funcionais lineares contínuos $\chi_{i} \in C_{i}^{*}, i=0, \ldots, \ell+1$, não todos nulos, que satisfazem a seguinte equação $\sum_{i=0}^{\ell+1} \chi_{i}=0$.

Observação 2.3. Com as condições do teorema anterior segue o seguinte resultado: Uma condição suficiente para assegurar $\chi_{0} \neq 0$ é que exista ao menos uma direção factível e uma direção tangente, isto é, $\cap_{i=1}^{\ell+1} C_{i} \neq \emptyset$.

A seguir consideremos o seguinte problema de controle ótimo discreto com restrições mistas:

$$
\begin{array}{cl}
\text { Minimizar } & \sum_{k=0}^{N} \psi_{k}\left(x_{k}, u_{k}\right) \\
\text { sujeito a } & x_{k+1}=f_{k}\left(x_{k}, u_{k}\right), \quad k=0, \ldots, N, \\
& b\left(x_{0}, x_{1}, \ldots, x_{N+1}, u_{0}, \ldots, u_{N}\right)=0, \quad \varphi\left(x_{0}, x_{N+1}\right)=0, \\
& g\left(x_{0}, x_{1}, \ldots, x_{N+1}, u_{0}, \ldots, u_{N}\right) \leq 0, \quad \phi\left(x_{0}, x_{N+1}\right) \leq 0,
\end{array}
$$

onde $\psi_{k}: \mathbb{R}^{n} \times \mathbb{R}^{m} \rightarrow \mathbb{R}, f_{k}: \mathbb{R}^{n} \times \mathbb{R}^{m} \rightarrow \mathbb{R}^{n}$, para todo $k=1, \ldots, N, b: \mathbb{R}^{n(N+2)} \times \mathbb{R}^{m(N+1)} \rightarrow \mathbb{R}^{r_{b}}$, $\varphi: \mathbb{R}^{n} \times \mathbb{R}^{n} \rightarrow \mathbb{R}^{r_{\varphi}}, g: \mathbb{R}^{n(N+2)} \times \mathbb{R}^{m(N+1)} \rightarrow \mathbb{R}^{r_{g}}, \phi: \mathbb{R}^{n} \times \mathbb{R}^{n} \rightarrow \mathbb{R}^{r_{\phi}}$, são funções continuamente diferenciáveis em relação a $x=\left(x_{0}, x_{1}, \ldots, x_{N+1}\right)$ e $u=\left(u_{0}, \ldots, u_{N}\right)$.

Definição 2.5. Um processo factível é um par $(x, u)$ que satisfaz as restrições do problema $\left(P_{2}\right)$. Além disso, dizemos que $\left(x^{*}, u^{*}\right)$ é um processo ótimo local se existe $\epsilon>0$ tal que

$$
\sum_{k=0}^{N} \psi_{k}\left(x_{k}^{*}, u_{k}^{*}\right) \leq \sum_{k=0}^{N} \psi_{k}\left(x_{k}, u_{k}\right),
$$

para todo processo factível $(x, u)$ que satisfaz

$$
\left\|x_{k}-x_{k}^{*}\right\|<\epsilon, k=0, \ldots, N+1,\left\|u_{k}-u_{k}^{*}\right\|<\epsilon, \quad k=0, \ldots, N .
$$

Para obter condições necessárias de primeira ordem para o problema $\left(P_{2}\right)$ usando o formalismo de Dubovitskii-Milyutin precisamos primeiro reescrever o problema $\left(P_{2}\right)$ da seguinte forma

$$
\begin{aligned}
\text { Minimizar } & F_{0}(x, u) \\
\text { sujeito a } & Q \cap V,
\end{aligned}
$$

onde $Q=Q_{E} \cap Q_{I}, Q_{E}=\left\{(x, u) \in \mathbb{R}^{n(N+2)} \times \mathbb{R}^{m(N+1)}: F_{E}(x, u)=0\right\}$, $Q_{I}=\left\{(x, u) \in \mathbb{R}^{n(N+2)} \times \mathbb{R}^{m(N+1)}: F_{I}(x, u) \leq 0\right\}, V$ é uma vizinhança da solução ótima, $F_{0}: \mathbb{R}^{n(N+2)} \times \mathbb{R}^{m(N+1)} \rightarrow \mathbb{R}$ é definido por $F_{0}(x, u)=\sum_{k=0}^{N} \psi_{k}\left(x_{k}, u_{k}\right)$, $F_{E}: \mathbb{R}^{n(N+2)} \times \mathbb{R}^{m(N+1)} \rightarrow \mathbb{R}^{n(N+2)} \times \mathbb{R}^{r_{\varphi}} \times \mathbb{R}^{r_{b}}$ é dado por

$$
F_{E}(x, u)=\left(x_{k+1}-f_{0}\left(x_{0}, u_{0}\right), \ldots, x_{N+1}-f_{N}\left(x_{N}, u_{N}\right), \varphi\left(x_{0}, x_{N+1}\right), b(x, u)\right),
$$


e $F_{I}: \mathbb{R}^{n(N+2)} \times \mathbb{R}^{m(N+1)} \rightarrow \times \mathbb{R}^{r_{\phi}} \times \mathbb{R}^{r_{g}}$ é definido como $F_{I}(x, u)=\left(\phi\left(x_{0}, x_{N+1}\right), g(x, u)\right)$. Dado um processo factível $\left(x^{*}, u^{*}\right)$, consideremos os seguintes conjuntos de índices: $I_{\phi}\left(x^{*}, u^{*}\right)=\{j \in$ $\left.\left\{1, \ldots, r_{\phi}\right\}: \phi_{j}\left(x^{*}, u^{*}\right)=0\right\}$ e $I_{g}\left(x^{*}, u^{*}\right)=\left\{j \in\left\{1, \ldots, r_{g}\right\}: g_{j}\left(x^{*}, u^{*}\right)=0\right\}$. Uma vez obtido o problema $\left(P_{3}\right)$, precisamos determinar, no processo ótimo $\left(x^{*}, u^{*}\right)$, o cone das restrições de descida da função objetivo (denotado por $D\left(F_{0},\left(x^{*}, u^{*}\right)\right)$ ), o cone das direções tangentes a $Q_{E}$ (denotado por $T\left(Q_{E},\left(x^{*}, u^{*}\right)\right)$ ) e o cone das direções factíveis a $Q_{I}\left(\right.$ denotado como $\left.V\left(Q_{I},\left(x^{*}, u^{*}\right)\right)\right)$, e seus cones duais denotados por $D\left(F_{0},\left(x^{*}, u^{*}\right)\right)^{*}, T\left(Q_{E},\left(x^{*}, u^{*}\right)\right)^{*}, V\left(Q_{I},\left(x^{*}, u^{*}\right)\right)^{*}$.

\section{Resultados Principais}

Usaremos uma condição de regularidade com a finalidade de obter condições não degeneradas no Princípio do Máximo Discreto a qual é mais forte que a regularidade dada em [8], porém mais fácil de manipular computacionalmente. Tal condição é definida da seguinte forma.

Definição 3.1. Seja $\left(x^{*}, u^{*}\right)$ um processo factivel. Dizemos que $\left(x^{*}, u^{*}\right)$ é um processo regular se existe uma vizinhança $V$ de $\left(x^{*}, u^{*}\right)$ tal que:

- $\nabla F_{E}(x, u)$ tem o mesmo posto para cada $(x, u) \in V$.

- Existe $(s, t) \in N u \nabla F_{E}\left(x^{*}, u^{*}\right), s=\left(s_{0}, \ldots, s_{N+1}\right), t=\left(t_{0}, \ldots, t_{N}\right)$ tal que

$$
\begin{aligned}
\nabla g_{j}\left(x^{*}, u^{*}\right)^{\top}(s, t)<0, & j \in I_{g}\left(x^{*}, u^{*}\right), \\
\nabla_{x_{0}} \phi_{j}\left(x^{*}, u^{*}\right)^{\top} s_{0}+\nabla_{x_{N+1}} \phi_{j}\left(x^{*}, u^{*}\right)^{\top} s_{N+1}<0, & j \in I_{\phi}\left(x^{*}, u^{*}\right),
\end{aligned}
$$

Definimos a função Hamiltoniana associada ao problema $\left(P_{2}\right)$

$$
H_{k}: \mathbb{R}^{n(N+2)} \times \mathbb{R}^{m(N+1)} \times \mathbb{R}^{n(N+1)} \times \mathbb{R} \times \mathbb{R}^{r_{b}} \times \mathbb{R}^{r_{g}} \rightarrow \mathbb{R},
$$

como $H_{k}(x, u, p, \xi, \lambda, \mu)=p_{k+1}^{\top} f_{k}\left(x_{k}, u_{k}\right)-\xi \psi_{k}\left(x_{k}, u_{k}\right)-\sum_{i=1}^{r_{b}} \lambda_{i} b_{i}(x, u)-\sum_{j=1}^{r_{g}} \mu_{j} g_{j}(x, u)$.

Teorema 3.1. Seja $\left(x^{*}, u^{*}\right)=\left(x_{0}^{*}, \ldots, x_{N+1}^{*}, u_{0}^{*}, \ldots, u_{N+1}^{*}\right)$ um processo ótimo local do problema $\left(P_{2}\right)$. Se $\left(x^{*}, u^{*}\right)$ é um processo regular, então existe $(p, \xi, \lambda, \gamma, \mu, \eta) \in \mathbb{R}^{n(N+1)} \times \mathbb{R} \times \mathbb{R}^{r_{b}} \times \mathbb{R}^{r_{\varphi}} \times$ $\mathbb{R}^{r_{g}} \times \mathbb{R}^{r_{\phi}}, \xi>0, \mu_{j} \geq 0, i=1, \ldots, r_{g}, \eta_{j} \geq 0, j=1, \ldots, r_{\phi}$, tais que as seguintes condições são satisfeitas:

(i) Equação adjunta:

$$
p_{k}=\nabla_{x_{k}} H_{k}\left(x^{*}, u^{*}, p_{k+1}, \lambda, \mu\right), \quad k=1, \ldots, N .
$$

(ii) Condição de transversalidade:

$$
\begin{aligned}
\nabla_{x_{0}} H_{0}\left(x^{*}, u^{*}, p_{1}, \lambda, \mu\right)= & \sum_{i=1}^{r_{\varphi}} \gamma_{i} \nabla_{x_{0}} \varphi_{i}\left(x_{0}^{*}, x_{N+1}^{*}\right)+\sum_{j=1}^{r_{\phi}} \eta_{j} \nabla_{x_{0}} \phi_{j}\left(x_{0}^{*}, x_{N+1}^{*}\right), \\
p_{N+1}=- & {\left[\sum_{i=1}^{r_{b}} \lambda_{i} \nabla_{x_{N+1}} b\left(x^{*}, u^{*}\right)+\sum_{j=1}^{r_{g}} \mu_{j} \nabla_{x_{N+1}} g\left(x^{*}, u^{*}\right)+\right.} \\
& \left.\sum_{i=1}^{r_{\varphi}} \gamma_{i} \nabla_{x_{N+1}} \varphi_{i}\left(x_{0}^{*}, x_{N+1}^{*}\right)+\sum_{j=1}^{r_{\phi}} \eta_{j} \nabla_{x_{N+1}} \phi_{j}\left(x_{0}^{*}, x_{N+1}^{*}\right)\right] .
\end{aligned}
$$




\section{(iii) Condição de estacionaridade:}

$$
\nabla_{u_{k}} H_{k}\left(x^{*}, u^{*}, p_{k+1}, \lambda, \mu\right)=0, \quad k=0, \ldots, N .
$$

(iv) Condição de complementaridade:

$$
\mu_{j} g_{j}\left(x^{*}, u^{*}\right)=0, \quad j=1, \ldots, r_{g}, \quad \eta_{j} \phi_{j}\left(x^{*}, u^{*}\right)=0, \quad j=1, \ldots, r_{\phi} .
$$

Ideia da prova. A caracterização dos cones $D\left(F_{0},\left(x^{*}, u^{*}\right)\right), V\left(Q_{I},\left(x^{*}, u^{*}\right)\right)$ e seus duais podem ser encontrados seguindo a mesma ideia usada em [3], mas para caracterizar o cone $T\left(Q_{E},\left(x^{*}, u^{*}\right)\right)$ será usada a condição de posto constante nas restrições de igualdade. Aqui utilizamos o Teorema 1 (ver [5]). Assim, obtemos os cones da seguinte forma:

$$
\begin{aligned}
& D\left(F_{0},\left(x^{*}, u^{*}\right)\right)=\left\{(s, t) \in \mathbb{R}^{n(N+2)} \times \mathbb{R}^{m(N+1)}: \sum_{k=0}^{N}\left(\nabla_{x_{k}} \psi_{k}\left(x_{k}^{*}, u_{k}^{*}\right)^{\top} s_{k}+\right.\right. \\
& \left.\left.\nabla_{u_{k}} \psi_{k}\left(x_{k}^{*}, u_{k}^{*}\right)^{\top} t_{k}\right)<0\right\} \\
& D\left(F_{0},\left(x^{*}, u^{*}\right)\right)^{*}=\left\{l_{0} \in\left(\mathbb{R}^{n(N+2)} \times \mathbb{R}^{m(N+1)}\right)^{*}: l_{0}(s, t)=-\xi \sum_{k=0}^{N}\left(\nabla_{x_{k}} \psi_{k}\left(x_{k}^{*}, u_{k}^{*}\right)^{\top} s_{k}+\right.\right. \\
& \left.\left.\nabla_{u_{k}} \psi_{k}\left(x_{k}^{*}, u_{k}^{*}\right)^{\top} t_{k}\right), \xi \geq 0\right\} \\
& V\left(Q_{I},\left(x^{*}, u^{*}\right)\right)=V\left(Q_{I_{1}}\left(x^{*}, u^{*}\right)\right) \bigcap V\left(Q_{I_{2}},\left(x^{*}, u^{*}\right)\right), \\
& V\left(Q_{I_{1}},\left(x^{*}, u^{*}\right)\right)=\bigcap_{j \in I_{\phi}\left(x^{*}, u^{*}\right)} V\left(Q_{I_{1}}^{j},\left(x^{*}, u^{*}\right)\right) ; \quad V\left(Q_{I_{2}},\left(x^{*}, u^{*}\right)\right)=\bigcap_{j \in I_{g}\left(x^{*}, u^{*}\right)} V\left(Q_{I_{2}}^{j},\left(x^{*}, u^{*}\right)\right), \\
& V\left(Q_{I_{1}}^{j},\left(x^{*}, u^{*}\right)\right)=\left\{(s, t) \in \mathbb{R}^{n(N+2)} \times \mathbb{R}^{m(N+1)}: \nabla_{x_{0}} \phi_{j}\left(x_{0}^{*}, x_{N+1}^{*}\right)^{\top} s_{0}+\right. \\
& \left.\nabla_{x_{N+1}} \phi_{j}\left(x_{0}^{*}, x_{N+1}^{*}\right)^{\top} s_{N+1}<0\right\}, j \in I_{\phi}\left(x^{*}, u^{*}\right), \\
& V\left(Q_{I_{2}}^{j},\left(x^{*}, u^{*}\right)\right)=\left\{(s, t) \in \mathbb{R}^{n(N+2)} \times \mathbb{R}^{m(N+1)}: \nabla_{x} g_{j}\left(x^{*}, u^{*}\right)^{\top} s+\right. \\
& \left.\nabla_{u} g_{j}\left(x^{*}, u^{*}\right)^{\top} t<0,\right\}, \quad j \in I_{g}\left(x^{*}, u^{*}\right) . \\
& V\left(Q_{I},\left(x^{*}, u^{*}\right)\right)^{*}=\left\{l_{2} \in\left(\mathbb{R}^{n(N+2)} \times \mathbb{R}^{m(N+1)}\right)^{*}:\right. \\
& l_{2}(s, t)=\sum_{j \in I_{\phi}\left(x^{*}, u^{*}\right)} \eta_{j}\left(\nabla_{x_{0}} \phi_{j}\left(x_{0}^{*}, x_{N+1}^{*}\right)^{\top} s_{0}+\nabla_{x_{N+1}} \phi_{j}\left(x_{0}^{*}, x_{N+1}^{*}\right)^{\top} s_{N+1}\right) \\
& \left.+\sum_{j \in I_{g}\left(x^{*}, u^{*}\right)} \mu_{j}\left(\nabla_{x} g_{j}\left(x^{*}, u^{*}\right)^{\top} s+\nabla_{u} g_{j}\left(x^{*}, u^{*}\right)^{\top} t\right)\right\} . \\
& T\left(Q_{E},\left(x^{*}, u^{*}\right)\right)=\mathrm{Nu} \nabla F_{E}\left(x^{*}, u^{*}\right) . \\
& T\left(Q_{E},\left(x^{*}, u^{*}\right)\right)^{*}=\left\{\bar{l}_{1} \in\left(\mathbb{R}^{n(N+2)} \times \mathbb{R}^{m(N+1)}\right)^{*}:\right. \\
& \bar{l}_{1}(s, t)=\sum_{k=0}^{N}\left\langle\bar{p}_{k+1}, \nabla_{x_{k}} f_{k}\left(x_{k}^{*}, u_{k}^{*}\right) s_{k}+\nabla_{u_{k}} f_{k}\left(x_{k}^{*}, u_{k}^{*}\right) t_{k}-s_{k+1}\right\rangle+ \\
& \left.\left\langle-\gamma, \nabla_{x_{0}} \varphi\left(x^{*}, u^{*}\right) s_{0}+\nabla_{x_{N+1}} \varphi\left(x^{*}, u^{*}\right) s_{N+1}\right\rangle+\left\langle-\lambda, \nabla b\left(x^{*}, u^{*}\right)(s, t)\right\rangle\right\} .
\end{aligned}
$$

Uma vez que foram determinados os cones e seus duais, se aplica o Teorema 2.1(DubovitskiiMilyutin), segue que existem $l_{0} \in D\left(F_{0},\left(x^{*}, u^{*}\right)\right)^{*}, l_{1} \in T\left(Q_{E},\left(x^{*}, u^{*}\right)\right)^{*}$ e $l_{2} \in V\left(Q_{I},\left(x^{*}, u^{*}\right)\right)^{*}$, não todos identicamente nulos, tais que $l_{0}+l_{1}+l_{2}=0$. Para cada $(s, t) \in \mathbb{R}^{n(N+2)} \times \mathbb{R}^{m(N+1)}$, temos $l_{0}(s, t)+l_{1}(s, t)+l_{2}(s, t)=0$. Logo, obtemos

$$
\begin{aligned}
& -\xi\left[\sum_{k=0}^{N}\left(\nabla_{x_{k}} \psi_{k}\left(x_{k}^{*}, u_{k}^{*}\right)^{\top} s_{k}+\nabla_{u_{k}} \psi_{k}\left(x_{k}^{*}, u_{k}^{*}\right)^{\top} t_{k}\right)\right]+\sum_{k=0}^{N}\left\langle-p_{k+1}, s_{k+1}-\nabla_{x_{k}} f_{k}\left(x_{k}^{*}, u_{k}^{*}\right) s_{k}-\right. \\
& \left.\nabla_{u_{k}} f_{k}\left(x_{k}^{*}, u_{k}^{*}\right) t_{k}\right\rangle+\left\langle-\lambda, \nabla b\left(x^{*}, u^{*}\right)(s, t)\right\rangle+\left\langle-\gamma, \nabla_{x_{0}} \varphi\left(x_{0}^{*}, x_{N+1}^{*}\right) s_{0}+\nabla_{x_{N+1}} \varphi\left(x_{0}^{*}, x_{N+1}^{*}\right) s_{N+1}\right\rangle \\
& +\left\langle-\mu, \nabla g\left(x^{*}, u^{*}\right)(s, t)\right\rangle+\left\langle-\eta, \nabla_{x_{0}} \phi\left(x_{0}^{*}, x_{N+1}^{*}\right) s_{0}+\nabla_{x_{N+1}} \phi\left(x_{0}^{*}, x_{N+1}^{*}\right) s_{N+1}\right\rangle=0 .
\end{aligned}
$$


Reordenando termos e aplicando as propriedades de operadores adjuntos, levando em conta que a equação anterior se cumpre para todo $(s, t) \in \mathbb{R}^{n(N+2)} \times \mathbb{R}^{m(N+1)}$, em particular se $(s, t)=(s, 0)$, e definindo $M=\left[\begin{array}{llll}M_{0} & M_{1} & \cdots & M_{N}+1\end{array}\right]$ e $s=\left[\begin{array}{llll}s_{0} & s_{1} & \cdots & s_{N+1}\end{array}\right]^{\top}$, onde

$$
\begin{aligned}
M_{0}= & \xi \nabla_{x_{0}} \psi_{0}\left(x_{0}^{*}, u_{0}^{*}\right)-\nabla_{x_{0}} f_{0}\left(x_{0}^{*}, u_{0}^{*}\right)^{\top} p_{1}+\nabla_{x_{0}} b\left(x^{*}, u^{*}\right)^{\top} \lambda+\nabla_{x_{0}} g\left(x^{*}, u^{*}\right)^{\top} \mu+ \\
& \nabla_{x_{0}} \varphi\left(x_{0}^{*}, x_{N+1}^{*}\right)^{\top} \gamma+\nabla_{x_{0}} \phi\left(x_{0}^{*}, x_{N+1}^{*}\right)^{\top} \eta, \\
M_{k}= & \xi \nabla_{x_{k}} \psi_{k}\left(x_{k}^{*}, u_{k}^{*}\right)+p_{k}-\nabla_{x_{k}} f_{k}\left(x_{k}^{*}, u_{k}^{*}\right)^{\top} p_{k+1}+\nabla_{x_{k}} b\left(x^{*}, u^{*}\right)^{\top} \lambda+ \\
& \nabla_{x_{k}} g\left(x^{*}, u^{*}\right)^{\top} \mu, \quad \text { para } k=1, \ldots, N, \\
M_{N+1}= & p_{N+1}+\nabla_{x_{N+1}} b\left(x^{*}, u^{*}\right)^{\top} \lambda+\nabla_{x_{N+1}} g\left(x^{*}, u^{*}\right)^{\top} \mu+\nabla_{x_{N+1}} \varphi\left(x_{0}^{*}, x_{N+1}^{*}\right)^{\top} \gamma+ \\
& \nabla_{x_{N+1}} \phi\left(x_{0}^{*}, x_{N+1}^{*}\right)^{\top} \eta,
\end{aligned}
$$

obtém-se que $M s=0$ para todo $s \in \mathbb{R}^{n(N+2)}$, de onde $M=0$. Isto significa que

$$
\begin{array}{r}
\nabla_{x_{0}} f_{0}\left(x_{0}^{*}, u_{0}^{*}\right)^{\top} p_{1}-\xi \nabla_{x_{0}} \psi_{0}\left(x_{0}^{*}, u_{0}^{*}\right)-\nabla_{x_{0}} b\left(x^{*}, u^{*}\right)^{\top} \lambda-\nabla_{x_{0}} g\left(x^{*}, u^{*}\right)^{\top} \mu=\nabla_{x_{0}} \varphi\left(x_{0}^{*}, x_{N+1}^{*}\right)^{\top} \gamma \\
+\nabla_{x_{0}} \phi\left(x_{0}^{*}, x_{N+1}^{*}\right)^{\top} \eta,
\end{array}
$$

Portanto, a equação adjunta e a condição de transversalidade são satisfeitas. De maneira análoga, considerando agora $(s, t)=(0, t)$ temos a condição de estacionaridade. A condição de complementaridade segue do fato que o conjunto das direções factíveis está associado com as restrições ativas. Por último, considerando a Observação 2.3 e a condição de regularidade (Definição 3.1) concluímos que o multiplicador $\xi \neq 0$. Assim o resultado é demonstrado.

A seguir apresentamos um exemplo que satisfaz as condições do teorema anterior.

\section{Exemplo 3.1.}

$$
\begin{array}{cl}
\text { Minimizar } & \left(x_{0}^{(1)}\right)^{2}+\left(x_{0}^{(2)}\right)^{2}+\left(u_{0}^{(1)}\right)^{2}+\left(u_{0}^{(2)}\right)^{2}+\left(u_{1}^{(1)}\right)^{2}+\left(u_{1}^{(2)}\right)^{2} \\
\text { sujeito a } & x_{1}^{(1)}=x_{0}^{(1)}+u_{0}^{(1)}, \quad x_{1}^{(2)}=x_{0}^{(2)}+u_{0}^{(2)}, \\
& x_{2}^{(1)}=x_{1}^{(1)}+u_{1}^{(1)}, \quad x_{2}^{(2)}=x_{1}^{(2)}+u_{1}^{(2)}, \\
& x_{0}^{(2)}+x_{1}^{(1)}+u_{0}^{(2)}+u_{1}^{(2)}=0, \quad x_{0}^{(1)}+x_{2}^{(2)}=0, \quad\left(x_{0}^{(1)}+x_{2}^{(2)}\right)^{2}=0, \\
& x_{0}^{(2)}-x_{1}^{(2)}-x_{2}^{(1)}-u_{0}^{(2)}-u_{1}^{(1)} \leq 0,
\end{array}
$$

onde $x_{k}=\left(x_{k}^{(1)}, x_{k}^{(2)}\right) \in \mathbb{R}^{2}, \quad k=0,1,2$, e $u_{k}=\left(u_{k}^{(1)}, u_{k}^{(2)}\right) \in \mathbb{R}^{2}, \quad k=0,1$.

Pode-se notar que $\left(x^{*}, u^{*}\right)=(0,0)$ é um processo ótimo e satisfaz a condição de regularidade, pois $\nabla F_{E}(x, u)$ tem posto constante 6 em uma vizinhança do processo factível $(0,0)$, onde

$$
\nabla F_{E}(x, u)=\left(\begin{array}{cccccccccc}
-1 & 0 & 1 & 0 & 0 & 0 & -1 & 0 & 0 & 0 \\
0 & -1 & 0 & 1 & 0 & 0 & 0 & -1 & 0 & 0 \\
0 & 0 & -1 & 0 & 1 & 0 & 0 & 0 & -1 & 0 \\
0 & 0 & 0 & -1 & 0 & 1 & 0 & 0 & 0 & -1 \\
1 & 0 & 0 & 0 & 0 & 1 & 0 & 0 & 0 & 0 \\
2\left(x_{0}^{(1)}+x_{2}^{(2)}\right) & 0 & 0 & 0 & 0 & 2\left(x_{0}^{(1)}+x_{2}^{(2)}\right) & 0 & 0 & 0 & 0 \\
0 & 1 & 1 & 0 & 0 & 0 & 0 & 1 & 0 & 1
\end{array}\right)
$$

Além disso, $N u \nabla F_{E}(0,0)=\operatorname{span}\left\{v_{1}, v_{2}, v_{3}, v_{4}\right\}$, onde $\quad v_{1}=(-1,1,-1,1,-1,1,0,0,0,0)^{\top}$, $v_{2}=(0,-1,0,0,0,0,0,1,0,0)^{\top}, v_{3}=(0,0,0,0,1,0,0,0,1,0)^{\top}, v_{4}=(0,-1,0,-1,0,0,0,0,0,1)^{\top}$. 
Assim, considerando $z=v_{2}+v_{3}=(0,-1,0,0,1,0,0,1,1,0)^{\top} \in N u \nabla F_{E}(0,0)$, como

$\nabla F_{I}(0,0)=(0,1,0,-1,-1,0,0,-1,-1,0)^{\top}$, segue que $\nabla F_{I}(0,0)^{\top} z=-4<0$. Assim, a Definição 3.1 está satisfeita. Logo, pelo Teorema 3.1, existe $(p, \xi, \gamma, \lambda, \mu) \in \mathbb{R}^{4} \times \mathbb{R} \times \mathbb{R} \times \mathbb{R} \times \mathbb{R}$, com $\xi \neq 0$, tal que as condições do Teorema 3.1 são satisfeitas, em particular podemos considerar $p=0, \xi=1, \lambda=0, \gamma=\left(\begin{array}{ll}0 & 1\end{array}\right)^{\top}$ e $\mu=0$.

\section{Conclusões}

Neste trabalho, obtivemos condições necessárias não degeneradas de primeira ordem para problemas de controle ótimo discreto via formalismo de Dubovitskii-Milyutin, usando uma condição de regularidade combinada tipo posto constante e Mangasarian-Fromovitz. Estas condições necessárias não degeneradas podem ser vistas como uma versão do princípio do máximo para problemas de controle ótimo discreto com restrições mistas.

\section{Agradecimentos}

J.F.M. Ascona agradece à CAPES pelo auxílio financeiro; R. Andreani recebeu apoio da FAPESP (processo 2013/07375-0); V.A. de Oliveira recebeu apoio da FAPESP (processo 2013/073750) e do CNPq (processo 305786/2018-0).

\section{Referências}

[1] Andreani, R., de Oliveira, V. A., Pereira, J. T. and Silva, G.N. A weak maximum principle for optimal control problems with mixed constraints under a constant rank condition, IMA J. Math. Control Inform., 37: 1021-1047, 2020. DOI:10.1093/imamci/dnz036.

[2] Boltyanskii, V. G. Optimal Control of Discrete Systems. Halsted Press, 1978.

[3] Girsanov, I. V. Lecture Notes in Economics and Mathematical Systems. Springer-Verlag, Berlin - Heidelberg - New York, volume 67, 1972.

[4] Ioffe, A. D. and Tihomirov, V. M. Theory of Extremal Problems. Amsterdam-New York, 1979.

[5] Minchenko, L. and Stakhovski, S. On relaxed constant rank regularity condition in mathematical programming, J. Optim., 60:429-440, 2011. DOI:10.1080/02331930902971377.

[6] Osmolovskii, N. P. and Veliov, V. M. Optimal control of agestructured systems with mixed state-control constraints, J. Math. Anal. Appl., 455:396-421, 2017. DOI:10.1016/j.jmaa.2017.05.069.

[7] Toan, N. T. and Thuy, L. Q. Second-order necessary optimality conditions for a discrete optimal control problem with mixed constraints, J. Glob. Optim., 64: 533-562, 2016. DOI:10.1007/s10898-015-0333-0.

[8] Rojas-Medar, M.A., Isoton, C., dos Santos, L.B. and Vivanco-Orellana V. Optimality conditions for discrete-time control problems, J. Optim. Theory Appl.,185:115-133, 2020. DOI: $10.1007 / \mathrm{s} 10957-020-01638-5$.

[9] Tu, P.N.V. Introductory Optimization Dynamics. Springer, Berlin, 1991. 